\title{
MERGHERS pilot: MeerKAT discovery of diffuse emission in nine massive Sunyaev-Zel'dovich-selected galaxy clusters from ACT
}

\author{
K. Knowles ${ }^{\circledR}, 1,2 \star$ D. S. Pillay, ${ }^{1,2}$ S. Amodeo, ${ }^{3}$ A. J. Baker, ${ }^{4}$ K. Basu, ${ }^{5}$ D. Crichton, ${ }^{6}$ F. de Gasperin ${ }^{\circledR}, 7,8$ \\ M. Devlin, ${ }^{9}$ C. Ferrari, ${ }^{10}$ M. Hilton, ${ }^{1,2}$ K. M. Huffenberger ${ }^{\circledR},{ }^{11}$ J. P. Hughes, ${ }^{4}$ B. J. Koopman, ${ }^{12}$ \\ K. Moodley, ${ }^{1,2}$ T. Mroczkowski, ${ }^{13}$ S. Naess, ${ }^{14}$ F. Nati, ${ }^{15}$ L. B. Newburgh, ${ }^{12}$ N. Oozeer ${ }^{\circledR}, 16,17$ L. Page, ${ }^{18}$ \\ B. Partridge,${ }^{19}$ C. Pfrommer ${ }^{\circledR},{ }^{20}$ M. Salatino, ${ }^{21,22}$ A. Schillaci, ${ }^{23}$ C. Sifón ${ }^{\circledR},{ }^{24}$ O. Smirnov, ${ }^{25,16}$ \\ S. P. Sikhosana, ${ }^{1,2}$ E. J. Wollack ${ }^{26}$ and Z. Xu ${ }^{\circledR 9,27}$ \\ Affiliations are listed at the end of the paper
}

Accepted 2021 March 27. Received 2021 March 23; in original form 2020 December 30

\begin{abstract}
The MeerKAT Exploration of Relics, Giant Halos, and Extragalactic Radio Sources (MERGHERS) survey is a planned project to study a large statistical sample of galaxy clusters with the MeerKAT observatory. Here we present the results of a 16-h pilot project, observed in response to the 2019 MeerKAT Shared Risk proposal call, to test the feasibility of using MeerKAT for a large cluster study using short $(0.2-2.1 \mathrm{~h})$ integration times. The pilot focuses on $1.28-\mathrm{GHz}$ observations of 13 massive, low-to-intermediate redshift $(0.22<z<0.65)$ clusters from the Sunyaev-Zel'dovich-selected Atacama Cosmology Telescope (ACT) DR5 catalogue that show multiwavelength indications of dynamical disturbance. With a 70 per cent detection rate (9/13 clusters), this pilot study validates our proposed MERGHERS observing strategy and provides twelve detections of diffuse emission, eleven of them new, indicating the strength of MeerKAT for such types of studies. The detections (signal-to-noise ratio $\gtrsim 6)$ are summarized as follows: two systems host both relic(s) and a giant radio halo, five systems host radio haloes, and two have candidate radio haloes. Power values, $k$-corrected to $1.4 \mathrm{GHz}$, assuming a fiducial spectral index of $\alpha=-1.3 \pm 0.4$, are consistent with known radio halo and relic scaling relations.
\end{abstract}

Key words: galaxies: clusters: general-radio continuum: general.

\section{INTRODUCTION}

In the last two decades, observations of diffuse, steep-spectrum, cluster-scale radio synchrotron emission have been used to study the physical link between the thermal and non-thermal components of the intracluster medium (ICM), constraining theories of cosmic ray transport and magnetic field evolution within the ICM (see reviews by Brunetti \& Jones 2014; van Weeren et al. 2019). A strong link has been found to the dynamics of the host cluster, with the largest diffuse emission classes such as radio haloes and relics found in massive, dynamically disturbed systems - radio haloes are thought to originate from merger-driven turbulence within the ICM, and relics have been related to the presence of cluster merger shocks or revived radio galaxy plasma (see e.g. van Weeren et al. 2019, and references therein). The radio halo and relic luminosities correlate with the host cluster's mass and thermal properties (Cassano et al. 2013; de Gasperin et al. 2014). However, cluster selection has been found to affect these relations: samples selected via their SunyaevZel'dovich (SZ; Sunyaev \& Zeldovich 1972) signal show a higher diffuse emission detection rate than X-ray-selected samples (Basu 2012; Sommer \& Basu 2014; Zandanel, Pfrommer \& Prada 2014; Bonafede et al. 2015; Cuciti et al. 2015), which may be due to an $\mathrm{X}$-ray selection bias towards relaxed, cool-core clusters (Andrade-

^E-mail: kendaknowles.astro@gmail.com
Santos et al. 2017), or the different time-scales of SZ versus X-ray signal boosting during mergers (Randall, Sarazin \& Ricker 2002; Poole et al. 2007; Wik et al. 2008).

All statistical, uniformly selected cluster samples used in radio halo studies have to date been restricted to high masses $\left(M_{500} \gtrsim 6 \times\right.$ $\left.10^{14} \mathrm{M}_{\odot}\right)$ and lower redshift ranges $(0.1 \lesssim z<0.4$; e.g. Venturi et al. 2007; Cuciti et al. 2015), with current turbulent re-acceleration formation theories predicting a sharp drop in the occurrence of radio haloes at higher redshift, where cluster magnetic fields are expected to be weaker (Cassano, Brunetti \& Setti 2006; Brunetti \& Jones 2014). Diffuse emission has also been detected in some low-mass (e.g. Bernardi et al. 2016; Knowles et al. 2016b; Kale et al. 2017; Brüggen et al. 2018; Hlavacek-Larrondo et al. 2018; Hoang et al. 2020) and high-redshift ( $z>0.5$; Bonafede et al. 2009; PandeyPommier et al. 2013; Lindner et al. 2014; Riseley et al. 2017; Knowles et al. 2019; Giovannini et al. 2020) systems. Most of these detections result from single-target programmes or small samples. A study of a large statistical sample that covers these extended mass and redshift ranges has not been carried out. Most recently, LOFAR imaging of a declination-selected sample of nineteen $z>0.6$, SZ-selected clusters revealed high-redshift diffuse emission with similar radio powers to those in lower redshift systems (Di Gennaro et al. 2020). This is an indication that our theories of the evolution of cosmic magnetic fields, and therefore of the production of diffuse emission, require further study. 
The next step in advancing our understanding of diffuse emission processes and imposing stronger constraints on formation models is to perform statistical studies on cluster samples that expand discovery space. Due to their much improved sensitivity, such a study has become possible using the new generation of Square Kilometre Array (SKA) precursor telescopes such as MeerKAT, LOFAR (van Haarlem et al. 2013), and ASKAP (Johnston et al. 2007). MeerKAT is currently the most sensitive instrument of its kind in the Southern hemisphere. This 64-element interferometer located in the South African Karoo region, described in Jonas \& MeerKAT Team (2016), Camilo et al. (2018), and Mauch et al. (2020), operates in the $S$ (1750 $3500 \mathrm{MHz}), L(900-1670 \mathrm{MHz})$, and $U H F(580-1015 \mathrm{MHz})$ bands. Its configuration makes it particularly well-suited to studies of diffuse cluster emission: its dense core, with $\sim 75$ percent of the antennas lying within a 1-km radius, provides superior brightness sensitivity to extended structures, while its outer ring antennas provide a maximum baseline of $8 \mathrm{~km}$ and therefore sufficient resolution to disentangle compact sources in all but the highest redshift targets.

Due to their redshift-independent selection functions, large-area SZ surveys are a more efficient way to select massive clusters at any redshift compared to X-ray surveys with telescopes like ROSAT (Voges 1993) or eROSITA (Cappelluti et al. 2011), and are therefore better suited to an expanded diffuse emission study. Large SZ cluster catalogues have been compiled by the Planck satellite (Planck Collaboration XXIX 2014; Planck Collaboration XXVII 2016), and the ground-based Atacama Cosmology Telescope (ACT; Hasselfield et al. 2013; Hilton et al. 2018) and South Pole Telescope (Bleem et al. 2015, 2020). Unlike the ground-based telescopes that have higher resolution, Planck misses clusters at low mass and at higher redshift due to beam dilution. ACT is undertaking its Advanced ACT (AdvACT; Henderson et al. 2016) survey of $\sim 18000 \mathrm{deg}^{2}$ of the Southern and Equatorial sky (Naess et al. 2020), with the first release of the cluster catalogue $\left(\mathrm{ACT} D \mathrm{DR} 5^{1}\right)$ containing more than 4000 optically confirmed clusters (Hilton et al. 2021).

The MERGHERS (MeerKAT Exploration of Relics, Giant Halos, and Extragalactic Radio Sources; Knowles et al. 2016a) survey is a planned large-scale radio follow-up of ACT cluster targets that will be blind to the cluster dynamical state and use short integration times on MeerKAT. We present here the results of a pilot study of 13 ACT DR5 clusters, to test the feasibility of using MeerKAT for a large-scale cluster programme such as MERGHERS.

This paper is organized as follows. In Section 2, we introduce the sample and discuss the observations. The data processing methodology and imaging are described in Section 3. Results are presented in Section 4, with concluding remarks in Section 6. In this paper, we adopt a $\Lambda$ CDM flat cosmology with $H_{0}=70 \mathrm{~km} \mathrm{~s}^{-1} \mathrm{Mpc}^{-1}, \Omega_{\mathrm{m}}=$ 0.3 , and $\Omega_{\Lambda}=0.7$.

\section{CLUSTER SAMPLE AND OBSERVING}

\subsection{Sample selection}

For our cluster selection, we used an early version of the ACT DR5 cluster catalogue based on the ACT data through 2016, and selected candidate massive mergers to ensure a high scientific return from the pilot project. The full MERGHERS sample will not be subject to this 'candidate merger' constraint, as it will be homogeneously mass-selected.

${ }^{1}$ The ACT DR5 catalogue uses the $\sim 13000 \mathrm{deg}^{2}$ away from the Galactic plane to search for clusters.
The selection criteria for the pilot sample were as follows. To ensure that our targets were robust cluster detections, we restricted the preliminary ACT DR5 sample to optically confirmed clusters ${ }^{2}$ with an $\mathrm{SZ}$ signal-to-noise ratio $(\mathrm{S} / \mathrm{N})$ greater than 10 , with a further Right Ascension (RA) range cut of $23^{\mathrm{h}}$ to $07^{\mathrm{h}}$. The RA restriction was based on an expected MeerKAT observing schedule ranging from July to November, and on our preference for nighttime observing. The $\mathrm{S} / \mathrm{N}$ cut ensures we selected high-mass systems, and we further constrained the selection to clusters with $z \lesssim 0.6$ that lie within the coverage of the Dark Energy Survey (DES; Abbott et al. 2018). These cuts reduced the sample pool to 29 clusters. To select potential mergers, we used a qualitative optical indication of disturbed morphology by calculating the positional offset between the SZ peak and the brightest cluster galaxy (BCG); the larger the offset, the more likely it is that the cluster is disturbed (Sehgal et al. 2013). We visually inspected the DES imaging to confirm the presence of multiple BCGs for clusters with significant or intermediate offsets.

The required integration time per target was determined through mock MeerKAT observations and a goal MeerKAT detection S/N of 10. The mock observations conservatively assumed $400 \mathrm{MHz}$ of useable bandwidth due to the known satellite-affected frequency ranges. The simulations assumed a halo detection with a surface brightness, given the cluster redshift and SZ-derived mass, ${ }^{3}$ determined from observed scaling relations (Cassano et al. 2013). Our final sample was selected so as to maximize the number of targets observed, while remaining, after observation overheads, within the 16-h time restriction of the 2019 MeerKAT Shared Risk proposal call. The list of 13 ACT DR5 clusters observed in this pilot study, along with the on-target times, is provided in Table 1 .

\subsection{Observations}

All data for this project were observed in MeerKAT's $L$-band receiver configuration, which has a native bandwidth of $856 \mathrm{MHz}$ and a central frequency of $1.28 \mathrm{GHz}$. We observed in full polarization using the 4096-channel mode and 8-s dump time. To make full use of the power of MeerKAT, all observations used at least 58 of the 64 antennas; we also required that at least seven of the nine outer ring antennas be included. This criterion ensured that we would retain sufficient resolution in the final images to disentangle compact sources from any observed diffuse emission: for the highest redshift cluster in our sample, $z=0.640$, a physical scale of $200 \mathrm{kpc}$ (a conservative lower limit for merger-related diffuse cluster emission) covers $\sim 29 \operatorname{arcsec}$ on the sky.

At $L$ band, a noise level of $10 \mu \mathrm{Jy}_{\text {beam }}{ }^{-1}$, using Briggs (Briggs 1995 ) robust 0 weighting for an $\sim 10$-arcsec beam, can be reached with less than an hour of MeerKAT time. ${ }^{4}$ This sensitivity makes MeerKAT a powerful tool for observing a large number of targets in a reasonable amount of telescope time. However, short integration times typically lead to poor $u v$-coverage and subsequently noisy point spread functions (PSFs). PSFs with complicated or bright sidelobes often create unwanted artefacts during image reconstruction, limiting the dynamic range.

\footnotetext{
${ }^{2}$ We refer the reader to Hilton et al. (2021) for the optical confirmation procedure.

${ }^{3}$ We use the weak-lensing-calibrated ACT masses.

${ }^{4}$ See the online MeerKAT sensitivity calculator at https://archive-gw-1.kat.a c.za/public/tools/continuum_sensitivity_calculator.html.
} 
Table 1. Cluster sample with observational and imaging details.

\begin{tabular}{|c|c|c|c|c|c|c|c|c|c|c|}
\hline $\begin{array}{l}(1) \\
\text { Name } \\
(\text { ACT-CL...) }\end{array}$ & $\begin{array}{c}(2) \\
\text { RA J2000 } \\
\left({ }^{\circ}\right)\end{array}$ & $\begin{array}{l}(3) \\
\text { Dec. J2000 } \\
\quad\left(^{\circ}\right)\end{array}$ & $\begin{array}{c}(4) \\
z\end{array}$ & $\begin{array}{c}(5) \\
M_{500} \\
\left(10^{14} \mathrm{M}_{\odot}\right)\end{array}$ & $\begin{array}{c}(6) \\
t_{\mathrm{src}} \\
(\mathrm{min})\end{array}$ & $\begin{array}{c}(7) \\
\text { Flagged } \\
\text { (per cent) }\end{array}$ & $\begin{array}{c}(8) \\
\sigma_{\mathrm{FR}} \\
\left(\mu \mathrm{Jy} \mathrm{beam}^{-1}\right)\end{array}$ & $\begin{array}{c}(9) \\
\theta_{\text {synth, FR }} \\
\left(\operatorname{arcmin}, \operatorname{arcsec},{ }^{\circ}\right)\end{array}$ & $\begin{array}{c}(10) \\
\sigma_{\mathrm{LS}} \\
\left(\mu \mathrm{Jy} \mathrm{beam}^{-1}\right)\end{array}$ & $\begin{array}{c}(11) \\
\theta_{\text {synth, LS }} \\
(\operatorname{arcsec})\end{array}$ \\
\hline J0013.3-4906 & 3.32748 & -49.11263 & 0.407 & 6.8 & 108 & 39.6 & 7.6 & $6.9,5.9,152.0$ & 24 & 21 \\
\hline $\mathrm{J} 0019.6+0336^{a}$ & 4.91085 & 3.60879 & 0.266 & 10.2 & 24 & 44.2 & 18.1 & $7.9,7.2,161.7$ & 52 & 21 \\
\hline $\mathrm{J} 0034.4+0225^{a}$ & 8.61022 & 2.42259 & 0.388 & 8.1 & 24 & 39.0 & 23.1 & $8.2,6.7,147.8$ & 77 & 26 \\
\hline $\mathrm{J} 0040.8-4407^{a}$ & 10.20664 & -44.13242 & 0.350 & 10.3 & 24 & 39.7 & 31.8 & $7.2,6.1,157.7$ & $260^{a}$ & 23 \\
\hline \multicolumn{11}{|c|}{ Epoch B - 58 antennas } \\
\hline J0106.1-0618 & 16.54119 & -6.31591 & 0.641 & 4.6 & 108 & 49.3 & 7.5 & $7.4,5.1,165.4$ & 25 & 23 \\
\hline J0159.0-3413 & 29.75418 & -34.22213 & 0.413 & 9.1 & 24 & 48.6 & 12.3 & $6.7,5.6,163.6$ & 35 & 21 \\
\hline $\mathrm{J} 0240.0+0115^{a}$ & 40.01278 & 1.26642 & 0.603 & 5.0 & 132 & 50.6 & 8.1 & $7.7,5.1,164.9$ & 27 & 23 \\
\hline \multicolumn{11}{|c|}{ Epoch $C-58$ antennas } \\
\hline J0245.5-5302 & 41.37543 & -53.03602 & 0.298 & 10.7 & 24 & 61.3 & 14.7 & $7.7,5.1,16.3$ & 39 & 23 \\
\hline
\end{tabular}

Notes. The clusters are listed by epoch of observation, with the number of antennas used in each epoch's observation. Columns: (1) ACT DR5 cluster name; (2) J2000 Right Ascension of the SZ peak; (3) J2000 Declination of the SZ peak; (4) cluster redshift; (5) ACT SZ weak-lensing-calibrated mass; (6) total MeerKAT time on target; (7) percentage of MeerKAT data flagged during processing, which includes the known frequency ranges affected by satellites; (8) full-resolution central rms noise; (9) full-resolution synthesized beam: major axis, minor axis, and position angle; (10-11) LS map rms noise and resolution, respectively - see Section 4 for details. ${ }^{a}$ Cluster field required direction-dependent corrections. Image has highly variable noise due to residual contamination by the bright source artefacts.

To mitigate the negative effects of short integration times, the total on-source time for each target was broken up into 12-min scans, each separated by approximately $1 \mathrm{~h}$. Targets with similar RA were grouped into epochs (or schedule blocks), with each epoch having a maximum RA range of $4 \mathrm{~h}$. There are three epochs in this pilot study, labelled A, B, and C, the groupings of which are listed in Table 1. Within a schedule block, scans of different targets were interleaved with each other, with a 2-min visit to an appropriate phase calibrator after each target scan. By grouping targets into RA-constrained schedule blocks, several targets could share a phase calibrator, reducing the calibration overhead for that epoch. As a flux and bandpass calibrator, PKS J1939-6342 or PMN J0408-6545 was observed for ten min at the beginning of each epoch, and again every $2 \mathrm{~h}$. Appendix A shows the $u v$-coverage from the final calibrated data from two target observations, with the effect of the chosen observing strategy clearly evident.

\section{DATA REDUCTION}

We made use of the OXKAT V1.0 $0^{5}$ software (Heywood 2020) to reduce and process the data for this project, with each epoch being processed separately. OXKAT is a semi-automated PYTHONbased reduction pipeline for MeerKAT data, currently optimized for $L$-band continuum observations. The reduction procedure makes use of several radio astronomy software packages, including CASA (McMullin et al. 2007) for cross-calibration and WSCLEAN ${ }^{6}$ (Offringa et al. 2014) for general imaging.

The OXKAT pipeline is currently automated up to directionindependent self-calibration (referred to as second-generation calibration or 2GC) and imaging, with the cross-calibration (or firstgeneration calibration, $1 \mathrm{GC}$ ) and initial imaging tasks run separately

\footnotetext{
${ }^{5}$ https://github.com/IanHeywood/oxkat.

${ }^{6} \mathrm{https} / / /$ sourceforge.net/p/wsclean/wiki/Home/.
}

from the 2GC steps. Third generation calibration (3GC), which implements direction-dependent corrections to the visibilities, is not automated, but still possible within the OXKAT framework. Here we detail the $1 \mathrm{GC}, 2 \mathrm{GC}$, and $3 \mathrm{GC}$ processing steps for our data, implemented using the OXKAT software. The total percentages of flagged data, mostly due to satellites and other radio frequency interference (RFI), and the final properties of the primary beamcorrected images are listed in Table 1.

\subsection{GC: cross-calibration and initial imaging}

All cross-calibration tasks, where known calibrator sources are used to correct the target phases and amplitudes, are carried out in CASA. The data are first averaged to 1024 channels and the field list is interrogated to determine primary calibrator, secondary calibrator, and target fields, as well as the target-secondary pairings. Initial flagging includes the known RFI-corrupted frequency bands (amounting to $\sim 40$ per cent of the original bandwidth, dominated by RFI from satellites) as well as any poorly performing antennas. This step is then followed by automatic flagging of the calibrators, before models of the secondary calibrator fields are created. Both the secondary and primary calibrator models are then used to correct the target fields, after which the calibrated target fields are split into individual measurement sets.

The corrected target data undergo automatic flagging before imaging with WSCLEAN, using the multifrequency and auto-masking options (Offringa \& Smirnov 2017) and a Briggs weighting of -0.3 . With a synthesized beam of $\sim 8$ arcsec, this weighting provides the best compromise between angular resolution and noise sensitivity for our data. To capture all sources visible within MeerKAT's field of view, we image a $3.1 \times 3.1 \mathrm{deg}^{2}$ region centred on the cluster target. This is $\sim 20$ per cent wider than the MeerKAT primary beam at the lowest $L$-band frequency. We also note that the effective observing frequency, $v_{0}$, of an image is dependent on the final flagged bandwidth, which varies across the three epochs. 


\subsection{GC: self-calibration}

Self-calibration of the target visibilities, where gain solutions derived using only target data are used to iteratively improve its calibration, is carried out using CASA and WSCLEAN. First, the initial mask from the 1GC target imaging step is used to re-image the corrected data: the mask tells the software where to look for true emission and an accurate mask can greatly improve image quality. The mask is updated during re-imaging, after which a target sky model is predicted. The sky model is then used in CASA to self-calibrate the target data, which determines a set of single-direction calibration solutions based on the phase centre of the data set. Finally, the 2GCcorrected data are re-imaged and the mask and sky model updated.

For most of our targets, 2GC improves the image quality by reducing artefacts and lowers the noise floor by $1.3-20.3$ percent, depending on the field, resulting in image qualities sufficient for our scientific purposes. However, in 4 of our 13 targets, namely $\mathrm{J} 0019.6+0336$, J0034.4+0225, J0240.0+0115, and J0248.2+0238, $2 \mathrm{GC}$ processing results in a noisier image with larger artefacts. In each of the four cases, the field is populated by several bright ( $>100 \mathrm{mJy}$ ), extended sources, spread out over the $\sim 9-\mathrm{deg}^{2}$ imaging field. In these cases, applying a calibration solution across the full field of view based only on the phase centre direction is insufficient and exacerbates amplitude errors around the brightest sources. For these fields, the measurement set is therefore reverted to the $1 \mathrm{GC}$ stage before carrying out direction-dependent corrections.

\subsection{GC: direction-dependent calibration}

For the four fields where $2 \mathrm{GC}$ processing resulted in a poorer quality

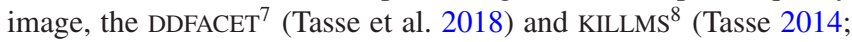
Smirnov \& Tasse 2015) packages are used to improve results through direction-dependent calibration. This is often needed for wide-field imaging where phase and amplitude gains may vary significantly across the field of view. With DDFACET and KILLMS, the imaging field is broken up into several regions, called facets, each with its own phase centre, typically a bright source. Calibration solutions are determined for each facet separately before being applied to the data. For our four data sets, the 1GC-corrected visibilities are reimaged with DDFACET, using between six and ten bright sources (depending on the field) to determine the facets. KILLMS uses these facets and source positions to determine phase and amplitude corrections on a per-facet basis. A second round of imaging in DDFACET applies these corrections on the fly to produce a $3 \mathrm{GC}$ image with reduced artefacts around bright sources. After $3 \mathrm{GC}$, the ACT-CL J0034.4+0225 field is still affected by direction-dependent artefacts. This field is particularly complex, with several bright (10$65 \mathrm{mJy}$ ), extended sources within the primary beam.

Additional calibration was also required for the J0040.8-4407 field, which is contaminated by a 2.6-Jy resolved source, $\sim 15$ arcmin from the pointing centre. Self-calibration improved the central rms noise by 20 per cent; however, the bright source was still a significant contaminant, with a central 2GC noise floor of $100 \mu \mathrm{Jy} \mathrm{beam}^{-1}$. To remove this bright source from the visibilities, we peeled the bright source using the $\mathrm{CUBICAL}^{9}$ package (Kenyon et al. 2018). The peeling process uses calibration solutions towards the direction of the interfering source to remove the source contributions from the visibilities before re-imaging. After peeling, the central rms noise

\footnotetext{
${ }^{7}$ https://github.com/saopicc/DDFacet.

${ }^{8}$ https://github.com/saopicc/killMS.

${ }^{9}$ https://github.com/ratt-ru/CubiCal.
}

level is improved by 69 percent to $31.8 \mu \mathrm{Jy}_{\text {beam }}{ }^{-1}$, and the peak image brightness is reduced to $0.11 \mathrm{Jy}$ beam ${ }^{-1}$. Additional processing of this field will be necessary to further reduce residual artefacts radiating from the peeled source region.

\subsection{Primary beam corrections}

Once a final image has been obtained, we use the KATBEAM ${ }^{10}$ package to create primary beam corrected images for analysis. The final images are masked such that they contain only regions where the primary beam response remains above 30 percent of the value at the phase centre. Primary beam correction increases the central rms noise by a median of 1.3 per cent across our 13 fields.

\section{RESULTS}

Table 1 lists the central rms noise, $\sigma_{\mathrm{FR}}$, and synthesized beam, $\theta_{\text {synth, FR }}$, for the final full-resolution, primary beam-corrected images. The native resolutions are in the range $6.7-8.3$ arcsec, with cluster region noise levels between 7.5 and $31.8 \mu \mathrm{Jy}_{\text {beam }}{ }^{-1}$.

To increase the sensitivity to faint extended emission, we need to image at lower resolution. However, extended structure would be contaminated by source blending if compact sources were not first removed. This step is typically taken using $u v$-range restricted source modeling and model-subtraction from the visibilities. This method failed on our datasets due to the combination of short integration times and MeerKAT's dense core: restricting the model to physical scales less than $100 \mathrm{kpc}^{11}$. our source model had too little data to accurately model the compact source flux; reducing the $u v$-cut to allow enough data for accurate flux characterization led to models that, when removed, led to oversubtraction of the extended emission. To image extended emission without compact sources, we instead implemented the image-plane filtering technique described in Rudnick (2002), whereby emission on scales one to three times the synthesized beam is filtered out, creating an image with only the larger scale emission. As no convolution takes place, the large-scale image has the same units and resolution as the original, but has a negatively offset zero-level that needs to be corrected before any quantitative analysis. After correction for this zero-level, the image is convolved, for aesthetics, with a Gaussian slightly larger than the filter size. To verify the success of the compact source removal, we checked the filtered maps (before convolving) at the positions of isolated compact sources and find values consistent with noise. The rms noise, $\sigma_{\mathrm{LS}}$, and convolved beam size, $\theta_{\text {synth, LS }}$, of the smoothed large-scale-emission images (hereafter the LS images) are given in the last two columns of Table 1 .

Fig. 1 shows the cluster regions for the nine systems in which we detect diffuse extended emission $(\mathrm{S} / \mathrm{N}>5)$, with two of the nine clusters containing more than one diffuse source. The fullresolution image is shown in the colour map, with the synthesized beam indicated by the filled red ellipse in the lower left-hand part of each panel. Black contours show the $[-3,3,5,10] \times \sigma_{\text {LS }}$ levels from the relevant LS map. Negative contours are dashed, and the LS synthesized beam is shown by the bold black ellipse in the lower lefthand part of each panel. The cross indicates the position of the ACT SZ peak. The physical scale at the cluster redshift is indicated in the upper left-hand part of each panel. Fig. B1 in the appendix shows the cluster regions for the four targets with no diffuse emission detection.

\footnotetext{
${ }^{10} \mathrm{https} / / /$ github.com/ska-sa/katbeam.

${ }^{11}$ The smallest types of diffuse emission are typically $100-400 \mathrm{kpc}$ across
} 

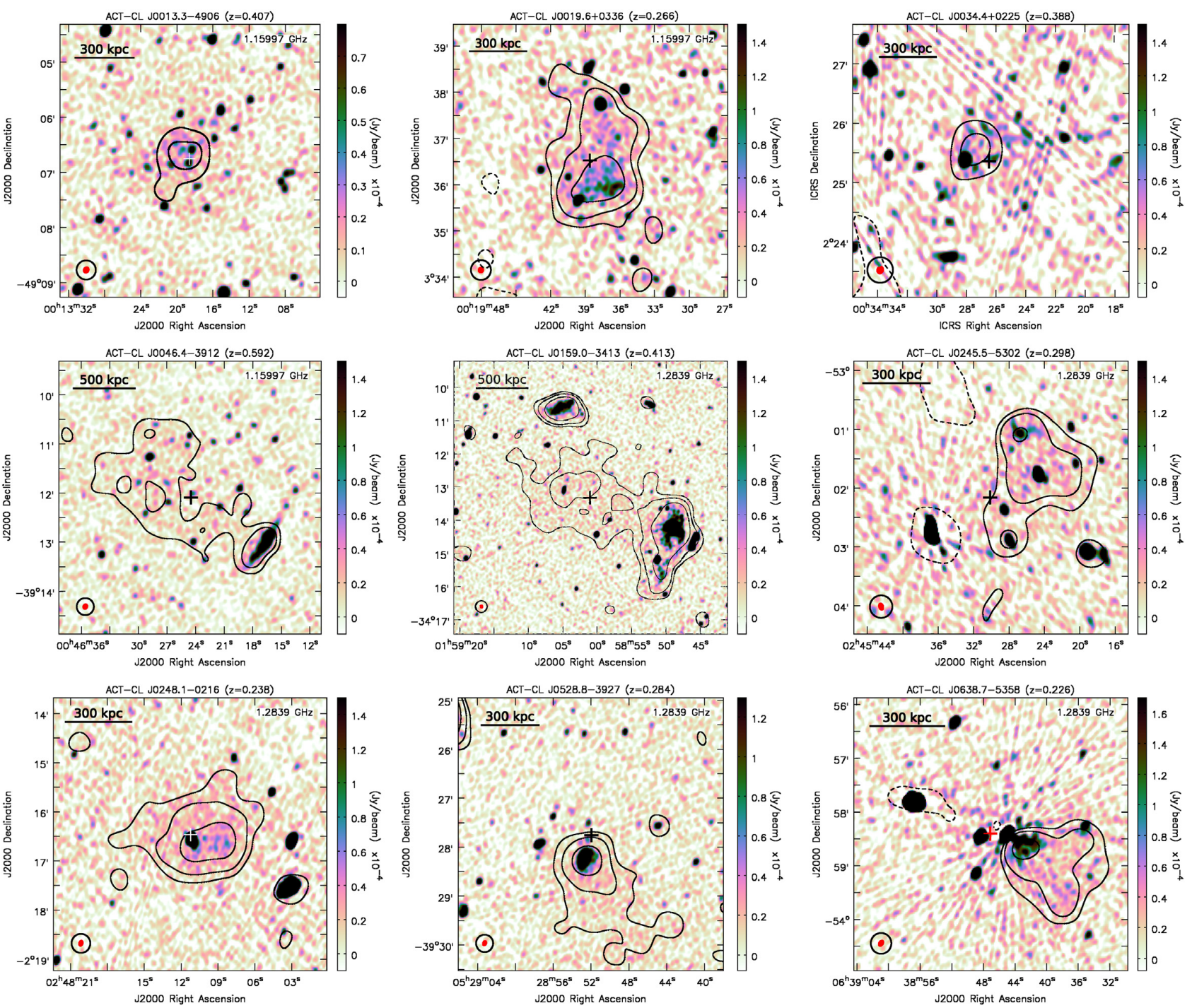

Figure 1. Full-resolution MeerKAT $L$-band images for the nine clusters with diffuse emission detections in our sample, with low-resolution contours from the LS map overlaid. In all panels, contours are at $[-3,3,5,10] \times \sigma_{\mathrm{LS}}$. Negative contours are dashed. The synthesized beam for both the full-resolution (filled red ellipse) and LS maps (open black circle) are indicated in the lower left-hand part of each panel. The beam sizes and central rms noise are provided in Table 1. The physical scale at the cluster redshift is indicated in the upper left-hand part of each panel, and the cross indicates the position of the ACT SZ peak. Artefacts remain in the ACT-CL J0034+0225 (top right-hand panel) and ACT-CL J0638.7-5358 (bottom right-hand panel) cluster fields; however, we are still able to recover extended emission in the cluster region.

We detect an $\sim 560$-kpc radio relic in ACT-CL J0046.4-3912, and double relics in ACT-CL J0159.0-3413. Sub-Mpc radio haloes are detected in five other systems, with candidate haloes in ACTCL J0013.3-4906 and ACT-CL J0034.4+0225. Given the observed sizes, the candidate haloes could also be mini-haloes, although unlikely if these are strongly dynamically disturbed systems. An $\mathrm{X}$-ray study of these systems may assist in a firmer classification. The LS maps confirm the full-resolution detections in all nine systems, and reveal giant radio haloes (physical size $>1 \mathrm{Mpc}$ ) in ACT-CL J0046.4-3912 and ACT-CL J0159.0-3413. Table 2 lists the largest angular size (LAS), largest projected physical size (LLS) at the cluster redshift, classification, effective observing frequency $v_{0}$, measured flux density $S_{v_{0}}$, and $\mathrm{S} / \mathrm{N}$ for each diffuse emission detection. Flux densities are measured within the $3 \sigma$ region and we assume a 5 per cent MeerKAT amplitude uncertainty. The total flux density uncertainty $\Delta S$ is measured as $\Delta S=\sqrt{(0.05 S)^{2}+N \sigma^{2}}$, where $N$ is the number of beams within the $3 \sigma$ region. With the exception of the radio relic in ACT-CL J0046.4-3912 and the northern relic in ACT-CL J0159.0-3413, which do not have embedded compact sources, all flux density measurements were made in the LS image. For the two former cases, the flux density is measured in the full-resolution image.

\section{DISCUSSION}

All but one of our detections are new, with the halo in ACTCL J0638.7-5358 having been detected by ASKAP (Wilber et al. 2020). We do not detect the full ASKAP halo emission in this system; however, we do resolve a peaked region in the halo that is morphologically aligned with the X-ray emission of the infalling subcluster seen in fig. 6 from Wilber et al. (2020).

To compare our detections to the literature, we determine $k$ corrected $1.4-\mathrm{GHz}$ radio powers, $P_{1.4 \mathrm{GHz}}$, for all diffuse emission 
Table 2. Measured properties of all diffuse emission detections in the sample.

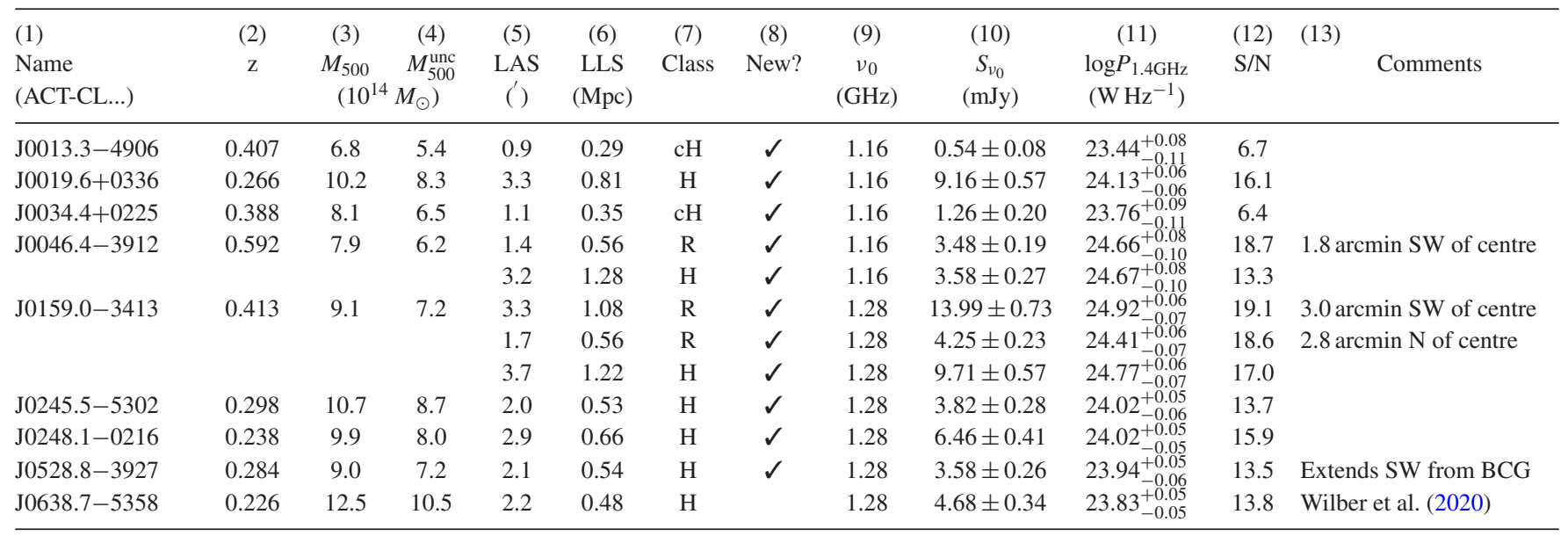

Notes. Columns: (1) ACT DR5 cluster name; (2) cluster redshift; (3) ACT SZ weak-lensing-calibrated mass; (4) ACT SZ uncalibrated mass; (5) largest angular size, in arcmin, of diffuse emission; (6) largest physical size, in Mpc, of diffuse emission at the cluster redshift; (7) classification: halo (H), relic (R), candidate (c); (8) new detection (or not); (9) effective observed frequency; (10) integrated flux density in mJy; (11) $k$-corrected radio power at $1.4 \mathrm{GHz}$, assuming $\alpha=$ $-1.3 \pm 0.4 ;(12) \mathrm{S} / \mathrm{N}$ of the MeerKAT detection; (13) comments.

detections. To extrapolate the MeerKAT flux densities to $1.4 \mathrm{GHz}$, we assume a fiducial spectral index of $\alpha=-1.3 \pm 0.4$, adopting the spectral power-law convention of $S_{v} \propto v^{\alpha}$. The choice of spectral index uncertainty allows for the wide range of observed spectral indices for radio haloes and relics. We note that the $k$-corrected radio powers will be higher if the detected sources have very steep spectra $(\alpha<-1.7)$; however, as the MeerKAT reference frequency is quite close to $1.4 \mathrm{GHz}$, the effect will not be large.

The calculated 1.4-GHz radio powers for all detections are provided in column 11 in Table 2, and we show their comparison with known scaling relations (Cuciti et al. 2021 for radio haloes; de Gasperin et al. 2014 for relics) in Fig. 2. We note that the literature results use SZ masses from Planck that have not been calibrated against weak lensing results, and are therefore systematically lower than our ACT DR5 masses used in selection. In Fig. 2 we therefore use the 'uncorrected' ACT DR5 SZ masses ${ }^{12}$ for our clusters, given in column 4 of Table 2, to more accurately compare results. All of our detections lie within the scatter of the correlations, indicating that we successfully removed contaminating compact emission. We note that our ACT-CL J0638.7-5358 radio halo, residing in the most massive of our clusters, lies at the edge of the scatter in the literature values, and in the region typically associated with ultrasteep spectrum sources. However, our power is likely underestimated due to missing flux, as described above, with Wilber et al. (2020) quoting a halo power a factor of $\sim 2.8$ higher, which would move it closer to the correlation.

\section{CONCLUSIONS}

The MERGHERS survey is a planned project to observe a statistically significant number $(\sim 200)$ of galaxy clusters with MeerKAT, in order to probe the cosmic and mass evolution of diffuse cluster radio emission and cosmic magnetic fields. Observing a statistically significant number of clusters in a single observing season requires relatively short $(\lesssim 1 \mathrm{~h}$ ) on-source times. We have presented the results of

${ }^{12}$ The 'uncorrected' ACT masses are referred to as $M_{500 \mathrm{c}}^{\mathrm{Unc}}$ in Hilton et al. (2021) and are in the M500CUNCORR columns of the ACT DR5 catalogue available on LAMBDA (https://lambda.gsfc.nasa.gov/product/act/actpol_pro d_table.cfm). a MERGHERS pilot study that validates this as feasible, confirming MeerKAT's suitability for large cluster studies at the $L$ band.

In this work, we have carried out pilot observations of 13 SZselected galaxy clusters detected in the ACT DR5 maps, observing each cluster for less than $2 \mathrm{~h}$, with most being observed for only $24 \mathrm{~min}$. Short observations can be susceptible to poor $u v$-coverage, which, in turn, negatively affects the point spread function and subsequently limits image quality. Our chosen observing strategy, to be implemented in the full MERGHERS programme, mitigates this risk by splitting a target's observation time into several smaller chunks, interspersed with observations of other clusters, so that a wider range of hour angle is covered for every target.

By mitigating sparse $u v$-coverage, and employing a range of modern data reduction and imaging techniques, we reached or improved upon the predicted rms noise floor, based on our total time-on-source, for most target fields. However, for the fields that underwent $3 \mathrm{GC}$ processing, our final rms noise is higher by a factor of 1.1-1.9 compared to the predicted value. This is likely because theoretical noise estimates do not take into account bright source contamination. For future observations, for fields with significant bright sources in all-sky surveys, full-field modelling may be a preferred method for estimating required source integration times. Due to its several extended bright sources, the ACT-CL J0034.4+0225 field is a good candidate for testing such algorithms, as well as testing more advanced reduction techniques.

We are able to reliably detect diffuse emission in 70 per cent of our clusters, with our highest redshift detection being at $z=0.592$. All but one of our detections are new and the full set, provided in Table 2, can be summarized as follows: Two systems host both radio relic(s) and a giant radio halo, five systems have radio haloes, and two have candidate radio haloes. Our estimated $1.4-\mathrm{GHz}$ radio powers for all detections, assuming a fiducial spectral index of $-1.3 \pm 0.4$, are consistent with known scaling relations. Determination of in-band spectral indices and power upper limits for the non-detections are outside of the scope of this detection paper. When combined with multiwavelength data to verify the cluster dynamical state, these radio results will allow us to study cluster magnetic fields out to $z \sim 0.6$.

Our MERGHERS pilot project has shown that MeerKAT's $L$ band provides sufficient resolution and sensitivity to reliably separate diffuse emission from compact sources. As MeerKAT also operates 

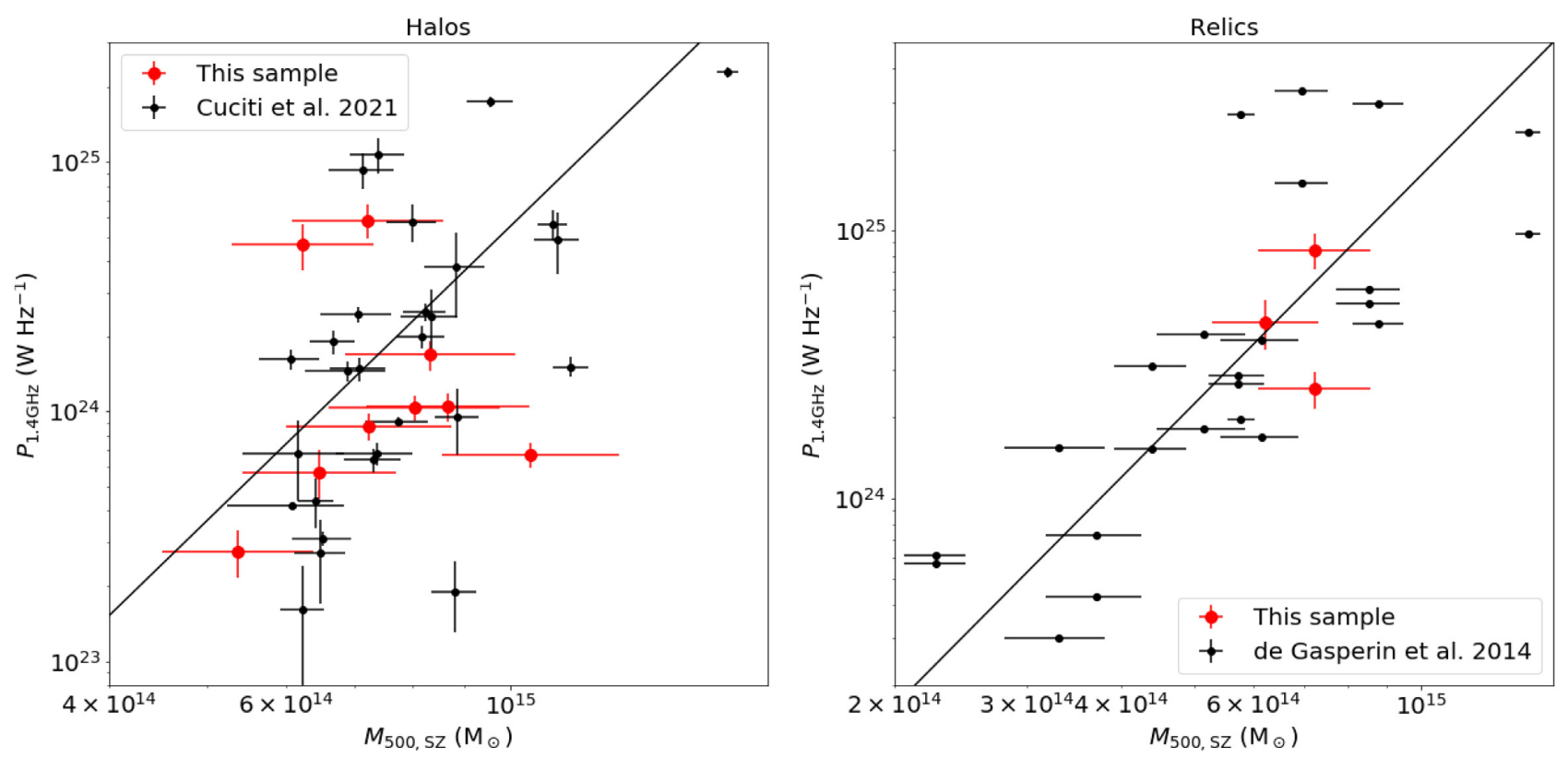

Figure 2. 1.4-GHz radio power versus $\mathrm{SZ} \mathrm{M}_{500}$ mass for the diffuse emission detections listed in Table 2 (large red points), compared to literature values (small black points). The literature values are from Planck (Planck Collaboration XXIX 2014;Planck Collaboration XXVII 2016) and we therefore use the uncorrected SZ masses from ACT DR5 to accurately compare our results. Left-hand panel: radio haloes in our sample, including candidates, compared with radio haloes from the statistical sample in Cuciti et al. (2021), where the solid line indicates their BCES Y|X best fit. Right-hand panel: radio relics in our sample compared with literature values from de Gasperin et al. (2014), with the solid line indicating their 'double + single relics' best fit.

at lower frequencies where the steep-spectrum diffuse emission is brighter, a similar test can be carried out with $U H F$-band data to determine the limiting redshift with the lower UHF resolution.

\section{ACKNOWLEDGEMENTS}

The MeerKAT telescope is operated by the South African Radio Astronomy Observatory (SARAO), which is a facility of the National Research Foundation, an agency of the Department of Science and Innovation.

KK, DP, and SS acknowledge funding support from SARAO. JPH acknowledges support from National Science Foundation Astronomy and Astrophysics Research Program award number 1615657. $\mathrm{MH}$ and KM acknowledge support from the National Research Foundation of South Africa. CP acknowledges support by the European Research Council under ERC-CoG grant CRAGSMAN646955. CS acknowledges support from the Agencia Nacional de Investigación y Desarrollo (ANID) through FONDECYT Iniciación grant no. 11191125. The research of OS is supported by the South African Research Chairs Initiative of the Department of Science and Technology and National Research Foundation. ZX is supported by theGordon and Betty Moore Foundation.

This research made use of the ASTROPY, ${ }^{13}$ (Astropy Collaboration et al. 2013, 2018), NUMPY (Harris et al. 2020), and SCIPY (Virtanen et al. 2020) PYTHON packages. ASTROPY is a community-developed core PYTHON package for astronomy. The Common Astronomy Software Applications (CASA) package is developed by an international consortium of scientists based at the National Radio Astronomical Observatory (NRAO), the European Southern Observatory (ESO), the National Astronomical Observatory of Japan (NAOJ), the Academia Sinica Institute of Astronomy and Astrophysics (ASIAA),

\footnotetext{
${ }^{13} \mathrm{http}: / /$ www.astropy.org.
}

the CSIRO division for Astronomy and Space Science (CASS), and the Netherlands Institute for Radio Astronomy (ASTRON) under the guidance of NRAO. The National Radio Astronomy Observatory is a facility of the National Science Foundation operated under cooperative agreement by Associated Universities, Inc.

\section{DATA AVAILABILITY}

The data underlying this paper will be shared on reasonable request to the corresponding author.

\section{REFERENCES}

Abbott T. M. C. et al., 2018, ApJS, 239, 18

Andrade-Santos F. et al., 2017, ApJ, 843, 76

Astropy Collaboration et al., 2013, A\&A, 558, A33

Astropy Collaboration et al., 2018, AJ, 156, 123

Basu K., 2012, MNRAS, 421, L112

Bernardi G. et al., 2016, MNRAS, 456, 1259

Bleem L. E. et al., 2015, ApJS, 216, 27

Bleem L. E. et al., 2020, ApJS, 247, 25

Bonafede A. et al., 2009, A\&A, 503, 707

Bonafede A. et al., 2015, MNRAS, 454, 3391

Briggs D. S., 1995, PhD thesis, New Mexico Institute of Mining and Technology

Brüggen M. et al., 2018, MNRAS, 477, 3461

Brunetti G., Jones T. W., 2014, Int. J. Mod. Phys. D, 23, 1430007

Camilo F . et al., 2018, ApJ, 856, 180

Cappelluti N. et al., 2011, Mem. Soc. Astron. Ital. Suppl., 17, 159

Cassano R. et al., 2013, ApJ, 777, 141

Cassano R., Brunetti G., Setti G., 2006, MNRAS, 369, 1577

Cuciti V. et al., 2021, A\&A, 647, A51

Cuciti V., Cassano R., Brunetti G., Dallacasa D., Kale R., Ettori S., Venturi T., 2015, A\&A, 580, A97

de Gasperin F., van Weeren R. J., Brüggen M., Vazza F., Bonafede A., Intema H. T., 2014, MNRAS, 444, 3130 
Di Gennaro G. et al., 2020, Nat. Astron., 5, 268

Giovannini G. et al., 2020, A\&A, 640, A108

Harris C. R. et al., 2020, Nature, 585, 357-362

Hasselfield M. et al., 2013, J. Cosmol. Astropart. Phys., 2013, 008

Henderson S. W. et al., 2016, J. Low Temp. Phys., 184, 772

Heywood I., 2020, Astrophysics Source Code Library, record ascl:2009.003

Hilton M. et al., 2018, ApJS, 235, 20

Hilton M. et al., 2021, ApJS, 253, 3

Hlavacek-Larrondo J. et al., 2018, MNRAS, 475, 2743

Hoang N. D. et al., 2020, MNRAS, 501, 576

Johnston S. et al., 2007, Publ. Astron. Soc. Aust., 24, 174

Jonas J., MeerKAT Team, 2016, MeerKAT Science: On the Pathway to the SKA

Kale R., Wik D. R., Giacintucci S., Venturi T., Brunetti G., Cassano R., Dallacasa D., de Gasperin F., 2017, MNRAS, 472, 940

Kenyon J. S., Smirnov O. M., Grobler T. L., Perkins S. J., 2018, MNRAS, 478, 2399

Knowles K. et al., 2016a, MeerKAT Science: On the Pathway to the SKA

Knowles K. et al., 2016b, MNRAS, 459, 4240

Knowles K. et al., 2019, MNRAS, 486, 1332

Lindner R. R. et al., 2014, ApJ, 786, 49

McMullin J. P., Waters B., Schiebel D., Young W., Golap K., 2007, in Shaw R. A., Hill F., Bell D. J., eds, ASP Conf. Ser. Vol. 376, Astronomical Data Analysis Software and Systems XVI. Astron. Soc. Pac., San Francisco, p. 127

Mauch T . et al., 2020, ApJ, 888, 61

Naess S. et al., 2020, J. Cosmol. Astropart. Phys., 2020, 046

Offringa A. R., Smirnov O., 2017, MNRAS, 471, 301

Offringa A. R. et al., 2014, MNRAS, 444, 606

Pandey-Pommier M., Richard J., Combes F., Dwarakanath K. S., Guiderdoni B., Ferrari C., Sirothia S., Narasimha D., 2013, A\&A, 557, A117

Planck CollaborationXXIX, 2014, A\&A, 571, A29

Planck Collaboration et al., 2016, A\&A, 594, A27

Poole G. B., Babul A., McCarthy I. G., Fardal M. A., Bildfell C. J., Quinn T., Mahdavi A., 2007, MNRAS, 380, 437

Randall S. W., Sarazin C. L., Ricker P. M., 2002, ApJ, 577, 579

Riseley C. J., Scaife A. M. M., Wise M. W., Clarke A. O., 2017, A\&A, 597, A96

Rudnick L., 2002, PASP, 114, 427

Sehgal N. et al., 2013, ApJ, 767, 38

Smirnov O. M., Tasse C., 2015, MNRAS, 449, 2668

Sommer M. W., Basu K., 2014, MNRAS, 437, 2163

Sunyaev R. A., Zeldovich Y. B., 1972, Comments Astrophys. Space Phys., 4, 173

Tasse C. et al., 2018, A\&A, 611, A87

Tasse C., 2014, A\&A, 566, A127

van Haarlem M. P. et al., 2013, A\&A, 556, A2

van Weeren R. J., de Gasperin F., Akamatsu H., Brüggen M., Feretti L., Kang H., Stroe A., Zandanel F., 2019, Space Sci. Rev., 215, 16

Venturi T., Giacintucci S., Brunetti G., Cassano R., Bardelli S., Dallacasa D., Setti G., 2007, A\&A, 463, 937

Virtanen P. et al., 2020, Nat. Methods, 17, 261

Voges W., 1993, Adv. Space Res., 13, 391

Wik D. R., Sarazin C. L., Ricker P. M., Randall S. W., 2008, ApJ, 680, 17

Wilber A. G., Johnston-Hollitt M., Duchesne S. W., Tasse C., Akamatsu H., Intema H., Hodgson T., 2020, PASA, 37, 40

Zandanel F., Pfrommer C., Prada F., 2014, MNRAS, 438, 124

\section{APPENDIX A: $U V$-COVERAGE PLOTS}

The observing strategy used in this project breaks up a target observation into 12-min scans and spreads these over a range of hour angles in order to improve the target $u v$-coverage. Fig. A1 presents the $u v$-coverage plots for one of our shortest observations (ACT-CL J0159.0-3413 - $24 \mathrm{~min}$; top panel) and for our longest observation (ACT-CL J0240.0+0115 - $132 \mathrm{~min}$; bottom panel). The plotted data are from the final calibrated data sets after all flagging.
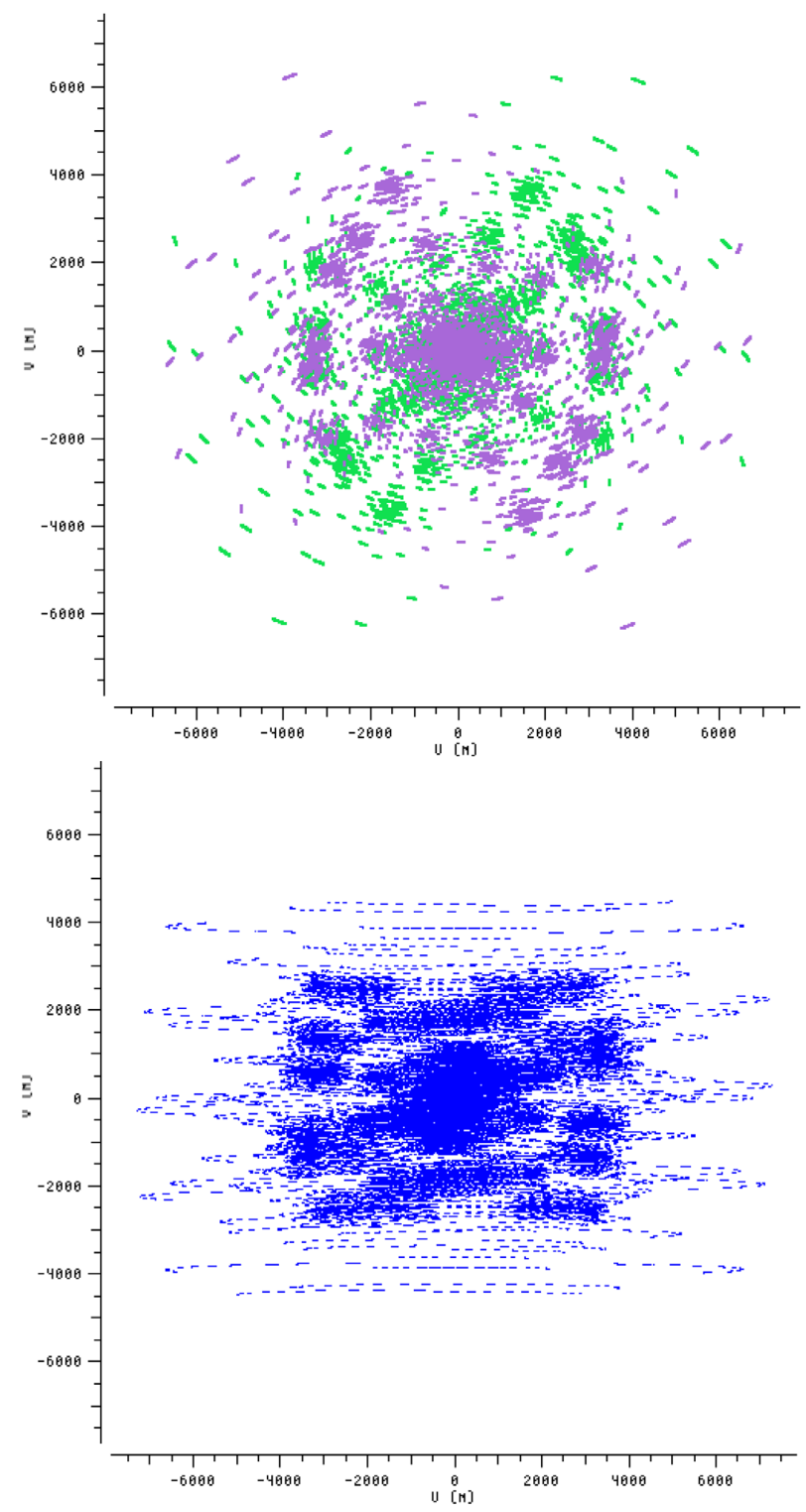

Figure A1. Target $u v$-coverage of the final calibrated data. The effect of MeerKAT's dense core is evident in the well-sampled 0-500 $\mathrm{m} u v$-range, providing maximal sensitivity to extended structure. Top panel: the 24-min observation of ACT-CL J0159.0-3413, with the two well-separated 12-min scans indicated by different colours. Bottom panel: our longest observation (132 min), targeting ACT-CL J0240.0+0115. The 'flattening' of the $u v$-tracks is due to the equatorial declination of the target.

The effect of spreading out shorter scans produces a wider coverage in the radial direction, as can be seen in the top panel where the two 12-min scans are in different colours. The effect of MeerKAT's dense core is evident in the well-sampled $0-500 \mathrm{~m}$ range, where there is maximal sensitivity to the extended emission we are searching for.

\section{APPENDIX B: CLUSTER FIELDS WITH NO DETECTIONS}

In Fig. B1, we present full-resolution images of the four targets in our sample with no diffuse emission detection in the cluster region. Colour scales and notations are as in Fig. 1. The targets are ACTCL J0040.8-4407 (top left-hand panel), ACT-CL J0106.1-0618 

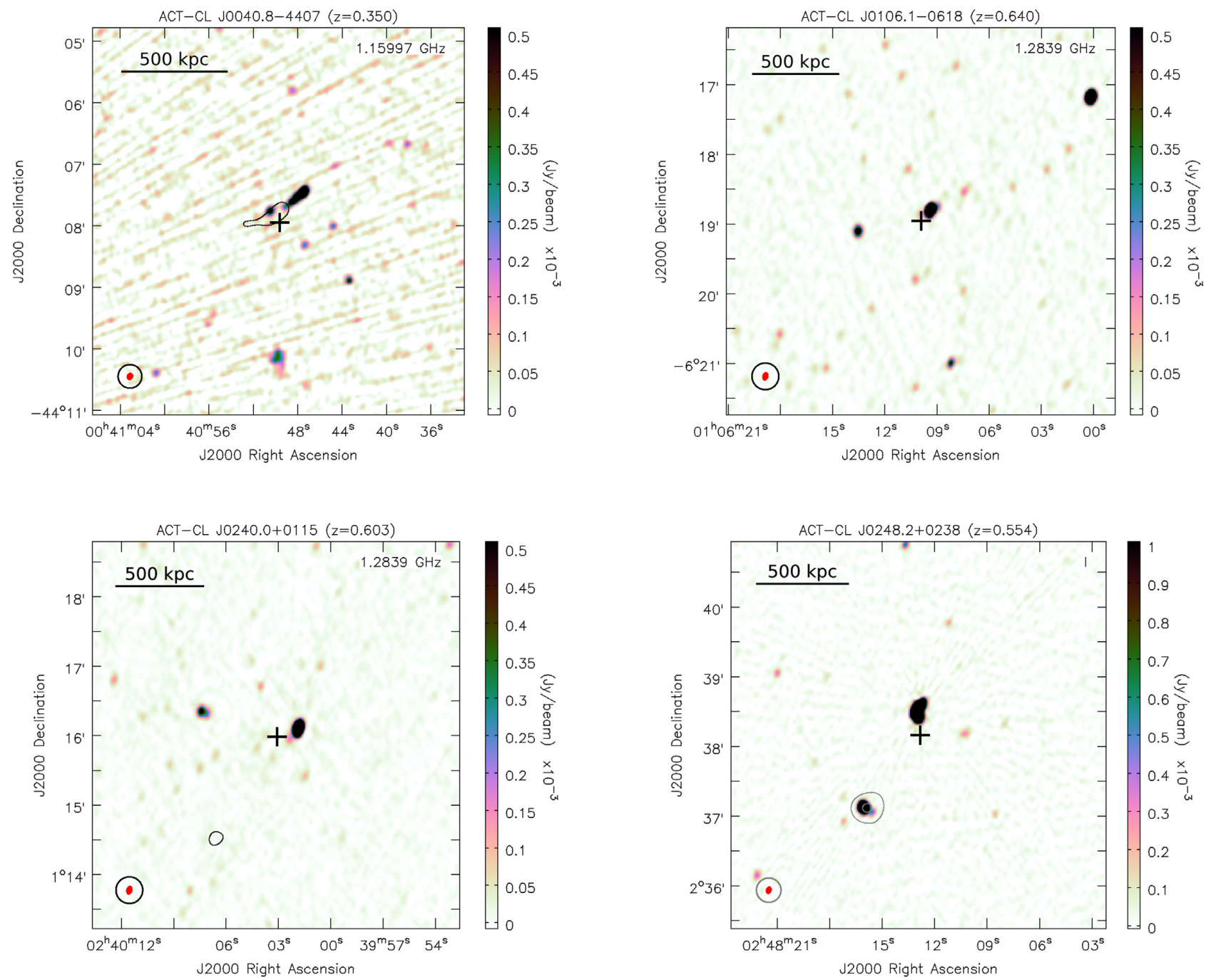

Figure B1. Full-resolution MeerKAT $L$-band images of the cluster region for the four targets in our sample with no diffuse emission detection. Contours are the $3 \sigma_{\text {LS }}$ level from the relevant LS map. The synthesized beam for both the full-resolution (filled red ellipse) and LS maps (open black circle) are indicated in the lower left-hand part of each panel. The beam sizes and central rms noise are provided in Table 1 . The physical scale at the cluster redshift is indicated in the upper left-hand part of each panel, and the cross indicates the position of the ACT SZ peak.

(top right-hand panel), ACT-CL J0240.0+0115 (bottom left-hand panel), and ACT-CL J0248.20+0238 (bottom right-hand panel).

\footnotetext{
${ }^{1}$ Astrophysics Research Centre, University of KwaZulu-Natal, Durban 4041, South Africa

${ }^{2}$ School of Mathematics, Statistics \& Computer Science, University of KwaZulu-Natal, Westville Campus, Durban 4041, South Africa

${ }^{3}$ Department of Astronomy, Cornell University, Ithaca, NY 14853, USA

${ }^{4}$ Department of Physics and Astronomy, Rutgers, the State University of New Jersey, 136 Frelinghuysen Rd., Piscataway, NJ 08854-8019, USA

${ }^{5}$ Argelander Institute for Astronomy, University of Bonn, Auf dem Huegel 71, D-53121 Bonn, Germany

${ }^{6}$ Institute for Particle Physics and Astrophysics, Eidgenössische Technische Hochschule Zürich, Wolfgang-Pauli-Str. 27, CH-8093 Zürich, Switzerland

${ }^{7}$ Hamburger Sternwarte, University of Hamburg, Gojenbergsweg 112, D21029 Hamburg, Germany

${ }^{8}$ Istituto di Radioastronomia, via P. Gobetti 101, I-40129 Bologna, Italy

${ }^{9}$ Department of Physics and Astronomy, University of Pennsylvania, 209 South 33rd Street, Philadelphia, PA 19104, USA

${ }^{10}$ Laboratoire Lagrange, Université Cote d'Azur, Observatoire de la Cote d'Azur, CNRS, F-06300 Nice, France
}

${ }^{11}$ Department of Physics, Florida State University, Tallahassee, FL 32306, USA

${ }^{12}$ Department of Physics, Yale University, New Haven, CT 06520, USA

${ }^{13}$ European Southern Observatory, Karl-Schwarzschild-Str. 2, D-85748 Garching b. München, Germany

${ }^{14}$ Center for Computational Astrophysics, Flatiron Institute, New York, NY 10010, USA

${ }^{15}$ Department of Physics, University of Milano-Bicocca, Piazza della Scienza 3, I-20126 Milano (MI), Italy

${ }^{16}$ South African Radio Astronomy Observatory, 2 Fir Street, Black River Park, Observatory, Cape Town 7925, South Africa

${ }^{17}$ African Institute for Mathematical Sciences, 6 Melrose Road, Muizenberg 7945, South Africa

${ }^{18}$ Joseph Henry Laboratories of Physics, Jadwin Hall, Princeton University, Princeton, NJ 08544, USA

${ }^{19}$ Department of Physics and Astronomy, Haverford College, Haverford, PA 19041, USA

${ }^{20}$ Leibniz-Institute for Astrophysics Potsdam (AIP), An der Sternwarte 16, D-14482 Potsdam, Germany

${ }^{21}$ Kavli Institute for Particle Astrophysics and Cosmology (KIPAC), Stanford, CA 94305, USA 
${ }^{22}$ Stanford University, Stanford, CA 94305, USA

${ }^{23}$ Department of Physics, California Institute of Technology, Pasadena, CA 91125, USA

${ }^{24}$ Instituto de Física, Pontificia Universidad Católica de Valparaíso, Casilla 4059, Valparaíso, Chile

${ }^{25}$ Department of Physics and Electronics, Rhodes University, PO Box 94, Makhanda 6140, South Africa
${ }^{26}$ NASA/Goddard Space Flight Center, 8800 Greenbelt Rd, Greenbelt, MD 20771, USA

${ }^{27}$ MIT Kavli Institute, Massachusetts Institute of Technology, Cambridge, MA, USA

This paper has been typeset from a $\mathrm{T}_{\mathrm{E}} \mathrm{X} / \mathrm{L} \mathrm{T}_{\mathrm{E}} \mathrm{X}$ file prepared by the author. 